\title{
Exact finite beam element for open thin walled doubly symmetric members under torsional and warping moments
}

\author{
Mohammed A. Hjaji *iD, Hasan M. Nagiar, Moftah M. Krar, Ezedine G. Allaboudi \\ University of Tripoli, Department of Mechanical and Industrial Engineering, Tripoli, Libya
}

\begin{abstract}
Starting with total potential energy variational principle, the governing equilibrium coupled equations for the torsional-warping static analysis of open thin-walled beams under various torsional and warping moments are derived. The formulation captures shear deformation effects due to warping. The exact closed form solutions for torsional rotation and warping deformation functions are then developed for the coupled system of two equations. The exact solutions are subsequently used to develop a family of shape functions which exactly satisfy the homogeneous form of the governing coupled equations. A super-convergent finite beam element is then formulated based on the exact shape functions. Key features of the beam element developed include its ability to (a) eliminate spatial discretization arising in commonly used finite elements, and (e) eliminate the need for time discretization. The results based on the present finite element solution are found to be in excellent agreement with those based on exact solution and ABAQUS finite beam element solution at a small fraction of the computational and modelling cost involved.
\end{abstract}

\section{Keywords}

Torsional-warping coupling; Exact shape functions; Warping moment; Exact beam element

Received: 16 August 2021; Accepted: 29 September 2021

ISSN: 2630-5763 (online) (C) 2021 Golden Light Publishing All rights reserved.

\section{Introduction}

Thin-walled members having open cross-sections are generally used as basic structural members in many engineering applications, as stiffeners in aircraft frame structures, in steel building structures, ship and marine structural frames and truck frames. In such applications, open thin-walled steel beams subjected to twisting moments prone to large normal warping stresses caused by the bimoments and excessive torsional rotation angles. Thus, it is important for the designers to avoid torsional moments in steel structures consisting of open thin-walled steel sections. However, in some practical applications, open thin-walled steel members subjected to twisting moments cannot be avoided and the designers will then need to calculate the magnitudes of torsional effects and to consider the torsional resistance of these thinwalled members.

For the thin-walled members with open crosssections under twisting moments, the cross-sections undergo longitudinal displacements and a plane section before deformation generally does not remain plain. This phenomenon is called warping. The Saint Venant torsion theory assumed that the warping is completely unrestrained, in which the torsional rotation angle per unit length remains constant along the structural member. In reality,

\footnotetext{
Corresponding author

Email: m.hjaji@uot.edu.ly
} 
many support details can prevent thin-walled member ends from warping freely. This causes an increase of the member torsional stiffness and introduces longitudinal stresses especially nearby the member ends. For solid and thin-wall closed sections these effects can be often ignored, while for open sections restrained warping can lead to significant longitudinal warping stresses. Neglecting these warping stresses may generate significant errors especially for open profile torsion beams.

Even though a large number of studies have been developed to investigate the static torsional response of open thin-walled doubly symmetric beams, to the best of the author's knowledge, no finite element solution based on exact shape functions have been reported for the static analysis of coupled torsional-warping of thin-walled doubly symmetric beams which account for shear deformation effects due to warping. Within the above context, the present study aims at developing an exact finite beam element solution for torsionalwarping coupled static response of thin-walled open beams with doubly symmetric cross-sections subjected to various twisting and warping moments.

\section{Literature review}

Thin-walled beam theories which capture warping effects include the works of Vlasov [1], Timoshenko [2] and Gjelsvik [3]. Vlasov [1] developed a general theory for isotropic thin-walled beams with open and closed sections which captures warping effects. Compared to the typical Saint Venant torsion theory, the Vlasov theory introduced the rate of change of the torsional rotation angle as a measure of warping deformation, which leads to an additional straining action, the bimoment. The Vlasov torsion formulation is based on two fundamental kinematic assumptions: (i) the cross section of a member remains undeformed (or rigid) after deformation, and (ii) the shear strain in the middle surface is neglected. In other words, Vlasov torsion theory for thin-walled beams considers the warping stiffness of the beam cross section but neglects the shear deformation effects at the middle surface. Timoshenko [2] independently developed a similar theory for isotropic beams with open cross-sections in which the shear deformation effects are included. Gjelsvik [3] extended the Vlasov's theory to account for the additional through-thickness secondary warping for beams with open and closed cross-sections.

There are well known analytical closed-form and finite element solutions for torsional static analysis of thin walled beams with open crosssection. Among them, Seaburg and Carter [4] derived the closed-form solutions for the torsional analysis of thin-walled beams under various twisting moments and boundary conditions. By including the transverse shear deformation and warping deformation, Back and Will [5] developed a finite element for the analysis of thin-walled beams with arbitrary open cross-sections. Mohareb and Nowzartash [6] developed a finite beam element formulation for torsional static analysis of thin-walled beams with open cross-sections based on St. Venant and Vlasov theories. Sapountzakis and Mokos [7] developed a boundary element solution for the general linear elastic non-uniform torsion problem of homogeneous and composite prismatic bars of arbitrary cross section subjected to various twisting moments. Kim and Kim [8] presented an improved thin-walled beam theory considering the transverse shear deformation due to the shearing force and restrained warping and the coupled effect between these two shear deformations by introducing Vlasov's assumption and applying Hellinger- Reissner principle. Pavazza [9] developed an analytical method for the torsion of open thin-walled beams with effect of shear deformation by assuming that the shear stress was constant along the beam length. Based on postulated stress field, Erkmen and Mohareb [10] developed a theory for the torsional static analysis of open steel thin-walled beams of general cross sections which accounted for shear deformation effects. El Fatmi [11,12] presented a beam theory with a non-uniform warping including the effects of torsion and shearing forces. Based on Vlasov's and Benscoter's theories, Campanile etal. [13] presented an exact solution of non-uniform torsion 
for thin-walled elastic beams with asymmetric cross-section. Based on the boundary element method, Mokos and Sapountzakis [14] developed a non-uniform torsion theory of doubly symmetrical arbitrary cross-section including secondary torsional moment deformation effect. Wang etal. [15] developed a first-order torsion theory based on Vlasov theory for restrained torsion of open thinwalled beams. The theory captured the warping deformation and restrained shear deformation of the cross-section. Sapountzakis [16] presented the static and dynamic analyses of the geometrically linear or nonlinear, elastic or elasto-plastic nonuniform torsion problems of bars of constant or variable arbitrary cross section subjected to arbitrarily distributed or concentrated twisting and warping moments along the bar axis. Based on the classical Vlasov's theory, Pavazza etal. [17] developed a theory for torsion of thin-walled beams with influence of shear deformation for open crosssections with single and double axes of symmetry and under various torsional loads. From SaintVenant and non-uniform torsional deformations, Aminbaghui etal. [18] formulated the governing differential equation for non-uniform torsion of thin-walled beams with open/closed cross-sections according to the theory of second-order torsional warping. Their formulation captured the effect of variable axial force and secondary torsion-moment deformation effect on the beam deformations due to torsional warping. In addition, the transfer matrix method is derived to develop a finite beam element with two nodes for static and dynamic analyses of beams. Mechab etal. [19] presented an analytical and numerical study of warping phenomenon of short composite thin-walled structural beams and thick plates based on the transverse shear higher order theory. Murin etal. [20] investigated the effect of torsional warping of thin-walled functionally graded material beams with doubly symmetric open and closed cross-sections. In their formulation, the longitudinal polynomial variation of the material properties and the secondary torsion moment on the eigen-frequencies were considered. The differential equations for Saint-Venant torsional deformations including the inertial line moments and were formulated. Considering the transfer functions, the solution of the differential equations is obtained using transfer matrix method to derive a local finite beam element with two-nodes for uniform and nonuniform torsion of beams. Aribas etal. [21] investigated the static and dynamic analyses of noncircular composite helical bars considering the exact helix geometry and warping effect. Nguyen etal. [22] developed a finite element method based on Vlasov theory to analyze the stress state induced due to bimoments of open thin-walled bars. Aribas etal. [23] investigated the transient dynamic response and stresses of composite elliptical and elliptical cone helices over exact axis geometry including warping effects using a mixed finite element method formulation. In their formulation, the transient response analysis is achieved using the Newmark time integration algorithm with/without the amplitude decay factor. According to the threedimensional elasticity theory, the constitutive equations of composite curved rods are derived. The mixed finite element method formulation is based on the first-order shear deformation theory and enhanced by a curvatures and displacementtype finite elements to investigate the influence of cross-sectional warping on the torsional rigidity and to evaluate the normal and shear stress distributions on the respective cross-sections of the composite curved rods.

A feature common to the above finite element studies is use of approximate shape functions involving spatial discretization errors, and thus requiring fine meshes to converge to the actual solution. In contrast, the present study avoids discretization errors by formulating exact shape functions which exactly satisfy the homogeneous solution of the governing equilibrium torsional static equations. Thus, the objective of this paper is to develop an accurate and efficient finite beam element solution which captures the torsionalwarping coupled static response of open thinwalled doubly symmetric members subjected to various twisting and warping moments. 


\section{Kinematic functions}

A straight uniform thin walled doubly symmetric cross-section beam of length $L$ is shown in Fig. 1. The thin-walled beam is referenced to a righthanded rectangular coordinates system $X, Y, Z$, where the axis $Z$ is the longitudinal axis of the beam, while $Y$ and $Z$ are the principal axes of the cross-section passing through the section centroid $C$. Another set of local coordinate system $(s, n, Z)$ is used, in which the coordinates $n$ and $s$ are taken along the normal and the tangent to the middle surface at the generic point $p(x, y)$ located on the mid-surface of the cross-section. The present theory is based on the following basic assumptions:

1. The formulation is applicable to thin-walled beams having doubly symmetric open crosssections.

2. The formulation is restricted to the torsional analysis of open section thin-walled beams.

3. Cross-section is assumed to remain perfectly rigid in its own plane throughout deformation,

4. The beam material is assumed to remain linearly elastic throughout deformation.

5. Displacements, strains and rotations are assumed to be small.

6. The beam cross-section is assumed to remain undeformed in its own plane in a manner consistent with Vlasov's first assumption (Vlasov [1]).

7. The shear deformation effects induced by warping (i.e., non-uniform torsion) at the middle surface of the cross-section are assumed non-zero and are characterized by a generalized displacement function multiplied by the sectorial coordinate.

According to the assumptions described above and by considering the doubly symmetric thin-walled beam to undergo torsional deformation only, the displacement functions $u_{p}(z, s), v_{p}(s, z)$ and $w_{p}(z, s)$ representing the torsional deformation of arbitrary point $p(x, y)$ are presented by:

$$
\begin{aligned}
& u_{p}(z, s)=-y(s) \theta_{z}(z) \\
& v_{p}(z, s)=x(s) \theta_{z}(z) \\
& w_{p}(z, s)=\omega(s) \psi(z)
\end{aligned}
$$

in which $\theta_{z}(z)$ is the torsional rotation, $x(s)$ and $y(s)$ are the coordinates of point $p(x, y)$ along the principal $X$ and $Y$ axes, $\psi(z)$ is a function which characterizes the magnitude of the warping deformation, $\omega(s)$ is the warping function of the open cross-section is defined by: $\omega(s)=\int_{s} h(s) d s$.
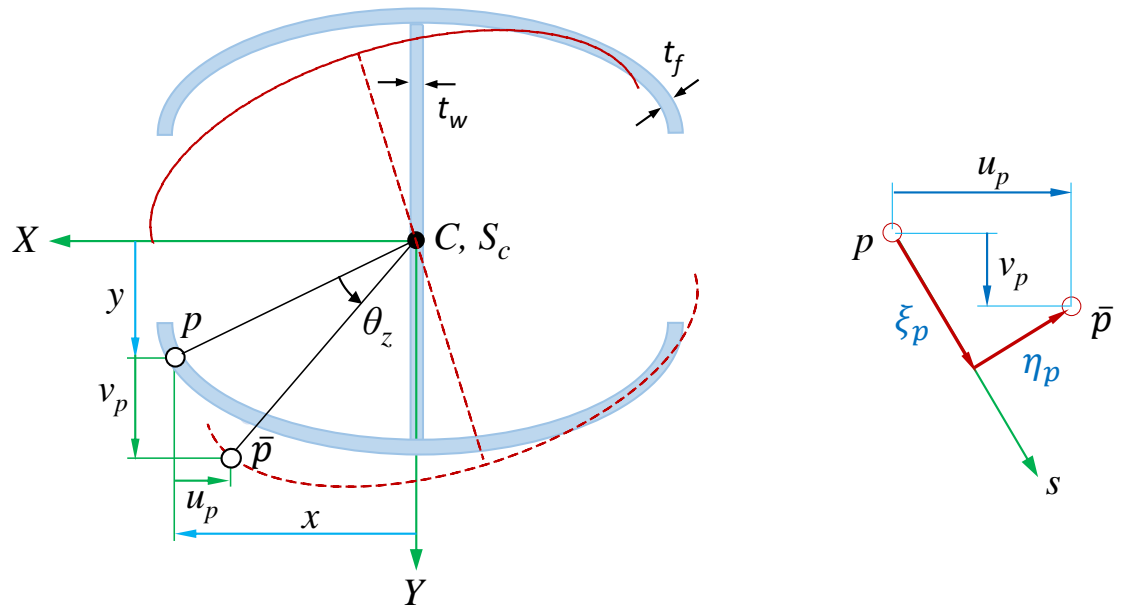

Fig. 1. Coordinate system and displacement components 
The in-plane lateral and transverse displacements $u_{p}(z, s)$ and $v_{p}(z, s)$ of the point $p(x, y)$ are resolved into tangential and normal displacement components $\xi_{p}(z, s)$ and $\eta_{p}(z, s)$ along the tangential and normal directions, respectively, yielding:

$$
\begin{aligned}
& \xi_{p}(z, s)=h(s) \theta_{z}(z, s) \\
& \eta_{p}(z, s)=r(s) \theta_{z}(z, s)
\end{aligned}
$$

where

$$
\begin{aligned}
& h(s)=x(s)(d y / d s)-y(s)(d x / d s) \\
& r(s)=x(s)(d x / d s)+y(s)(d y / d s)
\end{aligned}
$$

are the perpendicular distances from the shear center $S_{c}$ to the tangent and normal to the midsurface at point $p(x, y)$, respectively. The present study is focused on the linear response of thinwalled beams under small deformations, the nonzero axial and shear strains are then given as:

$$
\begin{aligned}
\varepsilon_{z z} & \approx \partial w_{p} / \partial z=\omega(s) \psi^{\prime}(z, t) \\
\gamma_{z s} & \approx\left(\partial w_{p} / \partial s\right)+\left(\partial \xi_{p} / \partial z\right) \\
& =h(s)\left[\psi(z, t)+\theta_{z}^{\prime}(z, t)\right]
\end{aligned}
$$

\section{Variational formulation}

The total potential energy $\Pi$ of thin-walled beam is calculated by the sum of elastic strain energy $U$ stored in the deformed beam and potential energy $V_{m}$ of the applied torsional and warping moments. Using the principle that the variation of the total potential energy is zero, the following statement is obtained as:

$$
\delta \Pi=\delta U+\delta V_{m}=0
$$

where $U$ is the internal strain energy defined as the sum of contributions of the normal stresses and St. Venant shear stresses, and is given by

$$
\begin{aligned}
\delta U= & \int_{0}^{\ell} \int_{A} E \varepsilon_{z z} \delta \varepsilon_{z z} d A d z+\int_{0}^{\ell} \int_{A} G \gamma_{z s} \delta \gamma_{z s} d A d z \\
& +\int_{0}^{\ell} G J \theta_{z}^{\prime} \delta \theta_{z}^{\prime} d z \\
= & \int_{0}^{L}\left[\left(G D_{\omega \omega}+G J\right) \theta_{z}^{\prime} \delta \theta_{z}^{\prime}+G D_{\omega \omega} \psi \delta \theta^{\prime}\right. \\
& \left.+E I_{\omega} \psi^{\prime} \delta \psi^{\prime}+G D_{\omega \omega}\left(\psi+\theta_{z}^{\prime}\right) \delta \psi\right] d z
\end{aligned}
$$

where $E$ is the modulus of elasticity, $G$ is the shear modulus, $J$ is the St. Venant torsional constant, and $A$ is the cross-sectional area. All primes denote derivatives with respect to space coordinate $z$. The variation of the potential energy $\delta V_{m}$ due to the applied torsional and warping moments is given by:

$$
\begin{aligned}
\delta V_{m} & =-\int_{0}^{L}\left[m_{z}(z) \theta_{z}(z)+m_{\omega}(z) \psi(z)\right] d z \\
& -\left[M_{z}(z) \delta \theta_{z}(z)\right]_{0}^{\ell}-\left[M_{\omega}(z) \delta \psi(z)\right]_{0}^{\ell}
\end{aligned}
$$

where $m_{z}(z)$ is the distributed twisting moment, $m_{\omega}(z)$ is the distributed bi-moment, and $M_{z}\left(z_{e}\right)$ is the concentrated twisting moments, $M_{\omega}\left(z_{e}\right)$ is the distributed bi-moment. The concentrated moments are applied at the beam ends (i.e., $z_{e}=0, L$ ).

\section{Equilibrium static torsional-warping equations}

From Eqs. (1-7) and by substituting into energy Eqs. (9-10), and the resulting expressions into Eq. (8), the governing coupled torsional-warping equilibrium static equations can be derived by integrating the derivatives of the varied quantities by parts and collecting the coefficients of $\delta \theta_{z}$ and $\delta \psi$, yields [24]:

$$
\begin{aligned}
& G\left(D_{\omega \omega}+J\right) \theta_{z}^{\prime \prime}(z)+G D_{\omega \omega} \psi^{\prime}(z)=m_{z}(z) \\
& G D_{\omega \omega} \theta_{z}^{\prime}(z)-E I_{\omega} \psi^{\prime \prime}(z)+G D_{\omega \omega} \psi(z)=m_{\omega}(z)
\end{aligned}
$$

The associated boundary conditions are obtained as:

$$
\left.\left[G\left(J+D_{\omega \omega}\right) \theta_{z}^{\prime}(z)+G D_{\omega \omega} \psi(z)-M_{z}(z)\right] \delta \theta_{z}(z)\right|_{0} ^{\ell}=0
$$




$$
\left.\left[E I_{\omega} \psi^{\prime}(z)+M_{\omega}(z)\right] \delta \psi(z)\right|_{0} ^{\ell}=0
$$

in which the sectional properties are defined by

$$
A, I_{x x}, I_{y y}, I_{\omega}, D_{\omega \omega}=\int_{A}\left[1, y^{2}, x^{2}, \omega^{2},(d \omega / d s)^{2}\right] d A
$$

Eqs. (11) and (12) are a set of coupled differential equations expressed in terms of the two dependent variables $\theta_{z}(z)$ and $\psi(z)$. By Eq. (11) for $\psi^{\prime}(z)$ and substituting the result in Eq. (12), a single fourth-order equation for torsional behavior of thinwalled doubly symmetric beams under uniform distributed twisting moment is obtained as:

$$
\frac{E I_{\omega}}{D_{\omega \omega}}\left(D_{\omega \omega}+J\right) \theta_{z}^{i v}(z)-G J \theta_{z}^{\prime \prime}(z)=-m_{z}(z)
$$

\subsection{Exact solution of torsional static equation}

The exact homogeneous solution of Eq. (15) is attained by setting the right-hand side of Eq. (15) equal to zero, i.e., $m_{z}(z)=0$, and assuming the torsional displacement $\theta_{z}(z)$ to take the following exponential form:

$$
\Theta_{z}(z)=A_{i} e^{m_{i} z}
$$

Substituting Eq. (16) into the homogeneous form of Eq. (15), the exact solution for torsional static behavior of open thin walled doubly symmetric I-beams under uniformly distributed torsional moment is then obtained as:

$\theta_{z}(z)=A+B z+C \cosh \beta z+D \sinh \beta z$

where $\beta=\sqrt{G J D_{\omega \omega} / E I_{\omega}\left(J+D_{\omega \omega}\right)}$.

By substituting Eq. (17) back into Eq. (12), the warping deformation function $\psi(z)$ is determined by

$$
\begin{aligned}
\psi(z) & =-B-\beta \sinh \beta z\left[1+\left(\frac{\lambda}{G D_{\omega \omega}}\right)\right] C \\
& -\beta \cosh \beta z\left[1+\left(\frac{\lambda}{G D_{\omega \omega}}\right)\right] D \\
& +\frac{m_{\omega}(z)}{G D_{\omega \omega}}-\frac{m_{z}(z)}{\left(G J-P_{z} r_{o}^{2}\right)} z
\end{aligned}
$$

where $\lambda=\frac{E I_{\omega}}{D_{\omega \omega}}\left(D_{\omega \omega}+J\right) \beta^{2}$.

The new Eq. (18) developed in this study is original for the literature used with Eq. (17) to obtain the exact shape functions for the present finite element formulation. Eq. (17) and (18) are rewritten in matrix form as:

$$
\{\Theta(z)\}_{2 \times 1}=[E(z)]_{2 \times 4}\{\bar{A}\}_{4 \times 1}
$$

in which

$$
[E(z)]=\left[\begin{array}{c:c:c:c}
1 & z & \cosh \beta z & \sinh \beta z \\
\hdashline 0 & -1 & -\beta\left[1+\left(\lambda / G D_{\omega \omega}\right)\right] \sinh \beta z & -\beta\left[1+\left(\lambda / G D_{\omega \omega}\right)\right] \cosh \beta z
\end{array}\right]
$$

where

$$
\begin{aligned}
& \langle\Theta(z)\rangle_{1 \times 2}=\left\langle\theta_{z}(z) \quad \psi(z)\right\rangle_{1 \times 2} \\
& \langle\bar{A}\rangle_{1 \times 4}=\langle A: B: C: D\rangle_{1 \times 4}
\end{aligned}
$$

\subsection{Finite element formulation}

A new finite beam element is developed for coupled torsional-warping static analysis of thin-walled beams under various torsional and warping moments. The proposed two-noded finite beam element having four degrees of freedom per element is developed. A set of exact shape functions that exactly satisfy the homogeneous solution of the coupled torsional-warping equations in (19) is used to formulate the exact stiffness matrix and load potential energy vector for the beam element.

\subsection{Formulation of exact shape function}

To relate the torsional rotation $\theta_{z}(z)$ and warping deformation $\psi(z)$ functions to the nodal torsional and warping deformation, the vector of integration constants $\{\bar{A}\}_{4 \times 1}$ is expressed in terms of nodal 
torsional and warping displacements $\left\langle S_{e}\right\rangle_{1 \times 4}=\left\langle\begin{array}{llll}\phi_{1} & \phi_{2} & \phi_{3} & \phi_{4}\end{array}\right\rangle_{1 \times 4}$ by enforcing the conditions $\theta(0)=\phi_{1}, \psi(0)=\phi_{2}, \theta(L)=\phi_{3}$ and $\psi(L)=\phi_{4}$, yields

$$
\begin{aligned}
& \left\{S_{e}\right\}_{4 \times 1}=\left\{\begin{array}{l}
\{\Theta(0)\}_{2 \times 1} \\
\{\Theta(L)\}_{2 \times 1}
\end{array}\right\}_{4 \times 1}=\left[\begin{array}{l}
{[E(0)]_{2 \times 4}} \\
{[E(L)]_{2 \times 4}}
\end{array}\right]_{4 \times 4} \\
& \{\bar{A}\}_{4 \times 1}=[\Phi]_{4 \times 4}\{\bar{A}\}_{4 \times 1}
\end{aligned}
$$

From Eq. (21), by substituting into Eq. (20), one obtains:

$$
\begin{aligned}
\{\Theta(z)\}_{2 \times 1} & =[E(z)]_{2 \times 4}[\Phi]_{4 \times 4}^{-1}\left\{S_{e}\right\}_{4 \times 1} \\
& =[H(z)]_{2 \times 4}\left\{S_{e}\right\}_{4 \times 1}
\end{aligned}
$$

in which

$$
\begin{aligned}
{[H(z)]_{2 \times 4} } & =\left[H_{1, j}(z): H_{2, j}(z)\right]_{2 \times 4} \\
& =[E(z)]_{2 \times 4}[\Phi]_{4 \times 4}^{-1}
\end{aligned}
$$

is a matrix of eight shape functions for torsional and warping deformation static behavior. It is seen that, Eq. (22) provided the exact shape functions that exactly satisfy the homogeneous solution of the torsional-warping static coupled equations are dependent on the beam length, and cross-sectional area.

\subsection{Energy expressions in terms of nodal torsional displacements}

The variation of strain energy and work done due to applied static torsional moments for open thinwalled beams are obtained in terms of nodal degrees of freedom substituting Eq. (22) into Eq. (9) as:

$$
\begin{aligned}
\delta U & =\left\langle\delta S_{e}\right\rangle_{1 \times 4}\left[\int _ { 0 } ^ { L } \left(\left[H^{\prime}(z)\right]_{4 \times 2}^{T}\left[Z_{m}\right]_{2 \times 2}\left[H^{\prime}(z)\right]_{2 \times 4}\right.\right. \\
& +[H(z)]_{4 \times 2}^{T}\left[Z_{c}\right]_{2 \times 2}\left[H_{c}(z)\right]_{2 \times 4} \\
& \left.\left.+\left[H_{b}(z)\right]_{4 \times 2}^{T}\left[Z_{b}\right]_{2 \times 2}[H(z)]_{2 \times 4}\right)\left\{S_{e}\right\}_{4 \times 1}\right] d z
\end{aligned}
$$

where

$$
\left[Z_{c}\right]_{2 \times 2}=\left[\begin{array}{cc}
0 & 0 \\
G D_{\omega \omega} & 0
\end{array}\right]_{2 \times 2},
$$

$\left[Z_{b}\right]_{2 \times 2}=\left[\begin{array}{cc}0 & 0 \\ 0 & G D_{\omega \omega}\end{array}\right]_{2 \times 2}$,

$\left[H_{c}(z)\right]_{4 \times 2}^{T}=\left[\begin{array}{ll}H_{1, j}^{\prime}(z) & H_{2, j}(z)\end{array}\right]_{4 \times 2}^{T}$,

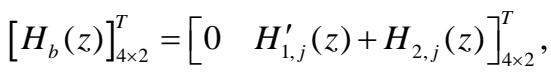

$\left[Z_{m}\right]_{2 \times 2}=\operatorname{diag}\left[G\left(D_{\omega \omega}+J\right) \quad E I_{\omega}\right]_{2 \times 2}$

The variation of the work done of the applied torsional loading presented by equation (10) can be given in terms of nodal degrees of freedom as:

$$
\begin{aligned}
\delta V_{m} & =-\left\langle\delta S_{e}\right\rangle_{1 \times 4}\left(\int_{0}^{L}[H(z)]_{4 \times 2}^{T}\left\{Q_{F}\right\}_{2 \times 1} d z\right. \\
& +\left[[H(z)]_{4 \times 2}^{T}\left\{Q_{m}\right\}_{2 \times 1}\right]_{0}^{L}
\end{aligned}
$$

where

$$
\begin{aligned}
& \left\langle Q_{F}\right\rangle_{1 \times 2}=\left\langle m_{z}(z) \quad m_{\omega}(z)\right\rangle_{1 \times 2}, \\
& \left\langle Q_{m}\right\rangle_{1 \times 2}=\left\langle\left[M_{z}(z)\right]_{0}^{L} \quad\left[M_{\omega}(z)\right]_{0}^{L}\right\rangle_{1 \times 2}
\end{aligned}
$$

\subsection{Matrix formulation}

From Eqs. (23-24), by substituting into Eq. (8), performing integration by parts, the element stiffness matrix $\left[k_{e}\right]_{4 \times 4}$ couples the nodal displacements $\left\{S_{e}\right\}_{4 \times 1}$ with corresponding nodal forces $\left\{F_{e}\right\}_{4 \times 1}$ is obtained as:

$$
\left[k_{e}\right]_{4 \times 4}\left\{S_{e}\right\}_{4 \times 1}=\left\{F_{e}\right\}_{4 \times 1}
$$

in which, the element stiffness matrix $\left[K_{e}\right]_{4 \times 4}$ is given by:

$$
\begin{aligned}
{\left[k_{e}\right]_{4 \times 4} } & =\left[H^{\prime}(z)\right]_{4 \times 2}^{T}\left[Z_{m}\right]_{2 \times 2}\left[H^{\prime}(z)\right]_{2 \times 4} \\
& +[H(z)]_{4 \times 2}^{T}\left[Z_{c}\right]_{2 \times 2}\left[H_{c}(z)\right]_{2 \times 4} \\
& +\left[H_{b}(z)\right]_{4 \times 2}^{T}\left[Z_{b}\right]_{2 \times 2}[H(z)]_{2 \times 4}
\end{aligned}
$$

The element load vector $\left\{F_{e}\right\}_{4 \times 1}$ is given by:

$$
\begin{aligned}
\left\{F_{e}\right\}_{4 \times 1} & =\int_{0}^{L}[H(z)]_{4 \times 2}^{T}\left\{Q_{F}\right\}_{2 \times 1} d z \\
& +\left[[H(z)]_{4 \times 2}^{T}\left\{Q_{m}\right\}_{2 \times 1}\right]_{0}^{L}
\end{aligned}
$$




\section{Numerical results and discussion}

In this section, several examples are presented to demonstrate the validity, accuracy and applicability of the finite beam element solution developed in the present study. The new finite beam element having two nodes and four degrees of freedom per element can capture the coupled static response for the torsional-warping analysis of open thin-walled beams under various torsional and warping moments. The present finite element formulation is based on the shape functions which exactly satisfy the exact solution of the coupled static field equations. This treatment eliminates mesh discretization errors arising in conventional interpolation schemes used in the finite element solutions and thus converge to the solution using a minimal number of degrees of freedom. As a result, it is observed that, the present numerical results obtained based on a new finite beam element using a single two-noded finite beam element per span yielded the corresponding results which exactly matched with those based on the exact closed-form solution provided in this study up to six significant digits. The results based on the present finite beam element (with two degrees of freedom per node) are compared with exact solution in [24] and ABAQUS finite beam BOS13 element (with seven degrees of freedom per node, i.e., three translations, three rotations and warping deformation). The numerical examples are investigated for doubly symmetric thin-walled beams with open cross-sections and a variety of torsional and warping moments and boundary conditions.

\subsection{Example 1-Cantilever I-beam under various twisting and warping moments}

A $3.0 \mathrm{~m}$ cantilever beam having open thin-walled doubly symmetric cross-section is subjected to $(i)$ concentrated twisting moment $M_{z}(L)=2.0 \mathrm{kN} . \mathrm{m}$ and warping moment $M_{\omega}(L)=0.8 \mathrm{kNm}^{2}$ applied at the cantilever free end (i.e., $z=L$ ), and (ii) uniformly distributed twisting moment $m_{z}(z)=1.2 \mathrm{kN} . \mathrm{m} / \mathrm{m}$ and distributed warping moment $m_{\omega}(z)=0.8 \mathrm{kNm}^{2} / \mathrm{m}$ applied along the beam axis as shown in Fig. 2. Geometrical properties of the doubly symmetric cross-section are given in Table 1. To validate the accuracy of the finite element formulation developed in this study, the static analyses of the coupled torsional-warping response of the cantilever thin-walled beam under the given concentrated and distributed twisting and warping moments are independently solved. The numerical results based on the present finite element formulation are compared to the corresponding results based on the exact closedform solution developed in previous study [24] and ABAQUS finite beam B31OS element solution.
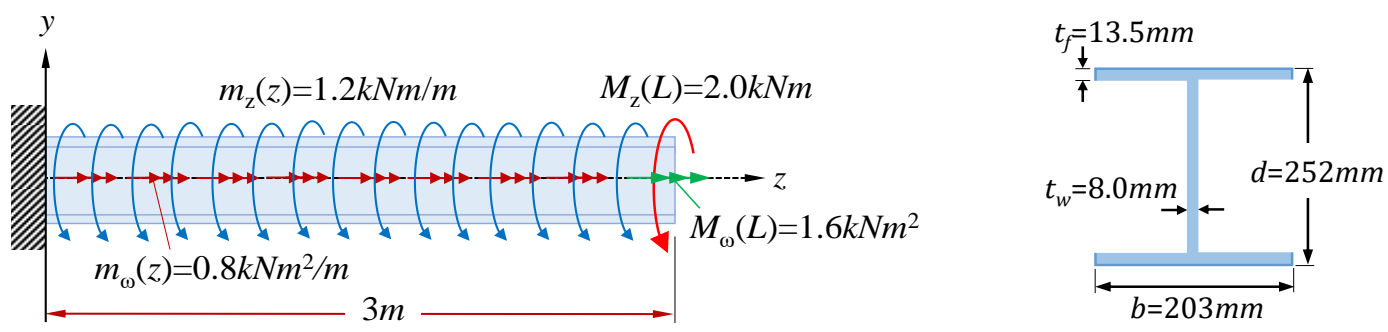

Fig. 2. A cantilever thin-walled I-beam under various twisting and warping moments 
Table 1. Geometric and properties of doubly symmetric thin-walled I-beam

\begin{tabular}{ll}
\hline E & $200 \times 10^{3} \mathrm{MPa}$ \\
$\mathrm{G}$ & $78 \times 10^{3} \mathrm{MPa}$ \\
$\mathrm{A}$ & $7420 \mathrm{~mm}^{2}$ \\
$\mathrm{I}_{\mathrm{xx}}$ & $87.10 \times 10^{6} \mathrm{~mm}^{4}$ \\
$\mathrm{I}_{\mathrm{yy}}$ & $18.82 \times 10^{6} \mathrm{~mm}^{4}$ \\
$\mathrm{~J}$ & $373.7 \times 10^{3} \mathrm{~mm}^{4}$ \\
$\mathrm{I}_{\omega}$ & $268.0 \times 10^{9} \mathrm{~mm}^{6}$ \\
$\mathrm{D}_{\omega \omega}$ & $77.94 \times 10^{6} \mathrm{~mm}^{4}$ \\
\hline
\end{tabular}

\subsubsection{Convergence analysis}

For the finite element model developed in the present finite beam element solution and ABAQUS finite beam B31OS element, various meshing sizes are considered to achieve the accuracy and validity of the numerical static results. To perform a convergence analysis, a cantilever thin-walled Ibeam subjected to uniformly distributed twisting moment is solved. In order to conform the static results for maximum torsional rotation $\theta_{z}(L)$ and warping deformation function $\psi(L)$ of the two finite element models; ABAQUS finite element model are refined as $20,40,60,80,100$ and 120 beam elements to yield the required accuracy, while the finite-element solution developed in the present study is based on exact shape functions and is conducted using a single finite beam element with two nodes and two degrees of freedom per node. Fig.3a-b shows the maximum torsional and

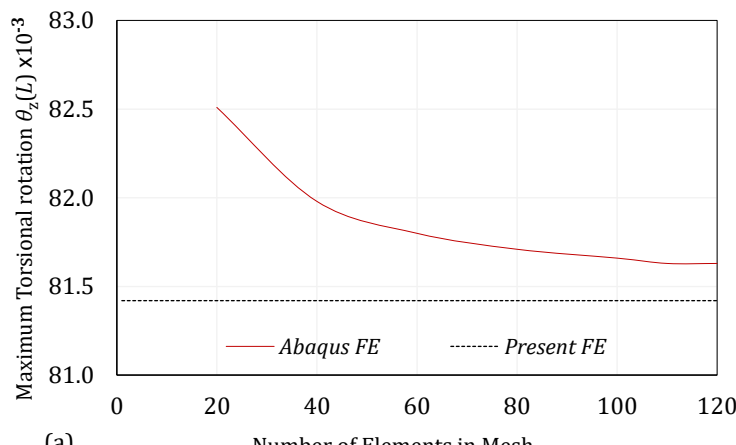

(a)

Fig. 3. Convergence analysis; (a) the maximum torsional rotation, and (b) the warping deformation function versus

Fig. 3. Convergence analysis; (a) the maximum torsional rotation, and
number of finite elements warping deformation static results, respectively, obtained from ABAQUS finite element model and present finite element versus number of finite elements. It is observed that, the torsional-warping results obtained from the finite element developed in the present study using one element are exactly matching with those based on the exact closed-form solution up to six significant digits. Whereas the maximum torsional-warping results obtained from ABAQUS model are gradually improved by increasing the number of finite beam elements. As illustrated from Fig. 3a-b, the differences between the ABAQUS finite element results and exact solution results are very small and the best match with the exact results are given in the ABAQUS model with 100 beam B31OS elements (i.e., seven degrees of freedom per node, a total of 707 DOFs in the model) to attain the accuracy.

Therefore, in ABAQUS finite element model solution, the beam is modelled using 100 beam B31OS elements along the longitudinal axis (i.e., 707 degrees of freedom) to eliminate the discretization errors and yield the accuracy of this example. Whereas the finite beam element solution developed in the present study is based on exact shape functions and is conducted using a single beam element with two nodes and four degrees of freedom. It was noted that solution based on a single finite beam element per span yielded results exactly matching those based on the closed-form solution provided in the previous study [24] up to six significant digits.

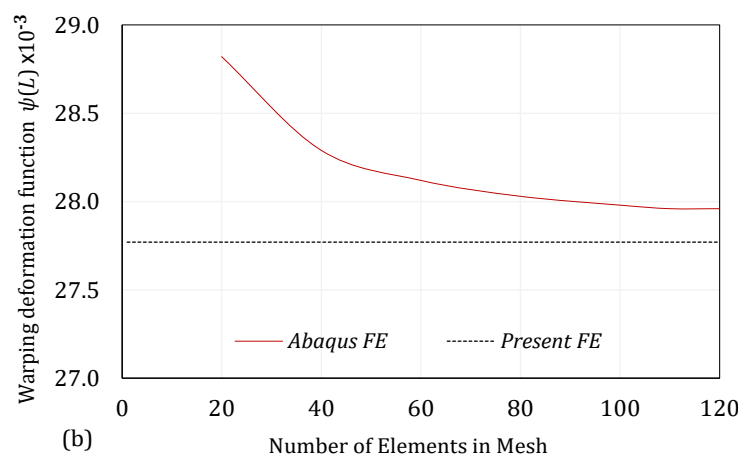

(b) Number of Elements in Mesh 
Table 2 provides the static results for coupled torsional-warping analysis obtained based on three different solutions: finite beam solution (FES) developed in the present study (which captures the shear deformation due to warping effects), the exact closed form solution presented in [24] and ABAQUS finite beam B31OS element solution (which capture only the shear deformation due to bending). It is observed that the finite element formulation developed in this study based on a single element (4 DOFs) are in excellent agreement with the results based on ABAQUS beam element solution using 100 B31OS beam elements (707 DOFs). This is a natural outcome of the fact that the present finite element solution is based on the shape functions which exactly satisfy the homogeneous form of the coupled torsional-warping static equations, which in turn eliminates discretization errors induced in the conventional finite element formulations.

Additionally, the static variation of torsional rotation angle $\theta_{z}(z)$ and warping deformation function $\psi(z)$ versus beam $\operatorname{span} z$ are illustrated in Figs. $4 a, c, e, g, i$ and $4 b, d, f, h, j$, respectively, for cantilever beam under various twisting and warping moments. The solutions, based on the exact closedform solution, ABAQUS finite beam B31OS element, and the present finite element solution are overlaid on the same diagrams for comparison. In the case of the ABAQUS finite element model, a total of $100 \mathrm{~B} 31 \mathrm{OS}$ beam elements (707 DOFs) were needed to achieve convergence, whereas the finite-element solution developed in the present study based on exact shape functions was conducted using a single finite element but for the sake of comparison 4 elements (10 DOFs) were used. It is obvious that, the present finite element formulation provides excellent agreement with exact closed form solution developed in [24] and ABAQUS beam model by keeping the number of degrees of freedom a minimum.

Table 2. Static results for torsional angle $\theta_{z}(L)$ and warping deformation function $\psi(L)$ at cantilever free end

\begin{tabular}{|c|c|c|c|c|c|}
\hline \multirow{2}{*}{ Type of load } & \multirow{2}{*}{$\begin{array}{l}\text { Function } \\
\text { type } \\
\left(\times 10^{-3}\right)\end{array}$} & \multirow{2}{*}{$\begin{array}{c}{[1]} \\
\text { Present FE } \\
\text { solution } \\
\text { (4 DOFs) }\end{array}$} & \multirow{2}{*}{$\begin{array}{c}{[2]} \\
\text { Exact CF } \\
\text { solution }\end{array}$} & \multirow{2}{*}{$\begin{array}{c}{[3]} \\
\text { ABAQUS } \\
\text { solution } \\
\text { (707 DOFs) }\end{array}$} & \multirow{2}{*}{$\begin{array}{c}\begin{array}{c}\text { Difference } \\
(\%)\end{array} \\
(3-1) / 3 \\
\end{array}$} \\
\hline & & & & & \\
\hline$\bigcap_{\mathrm{z}}^{M_{2}(L)}$ & $\theta_{\mathrm{z}}(L)$ & 115.2 & 115.2 & 115.3 & $0.09 \%$ \\
\hline$L$ & $\psi(L)$ & 53.70 & 53.70 & 53.72 & $0.04 \%$ \\
\hline $\begin{array}{l}y_{1} \\
y\end{array}$ & $\theta_{\mathrm{z}}(L)$ & 81.42 & 81.42 & 81.66 & $0.29 \%$ \\
\hline${ }_{L \longrightarrow}^{\operatorname{CocGCGG6GGS}}-z$ & $\psi(L)$ & 27.77 & 27.77 & 27.98 & $0.75 \%$ \\
\hline$M_{\omega}(L)$ & $\theta_{\mathrm{z}}(L)$ & 42.96 & 42.96 & 42.98 & $0.05 \%$ \\
\hline$\longrightarrow L \longrightarrow{ }^{2}$ & $\psi(L)$ & 39.61 & 39.61 & 39.63 & $0.05 \%$ \\
\hline$m_{\omega}(z)$ & $\theta_{\mathrm{z}}(L)$ & 45.72 & 45.72 & 45.78 & $0.13 \%$ \\
\hline L & $\psi(L)$ & 21.58 & 21.58 & 21.69 & $0.51 \%$ \\
\hline$m_{1}(z) \quad M_{z}(L)$ & $\theta_{\mathrm{z}}(L)$ & 285.5 & 285.5 & 285.7 & $0.07 \%$ \\
\hline$m_{\omega}(z)$ & $\psi(L)$ & 142.7 & 142.7 & 143.0 & $0.21 \%$ \\
\hline
\end{tabular}




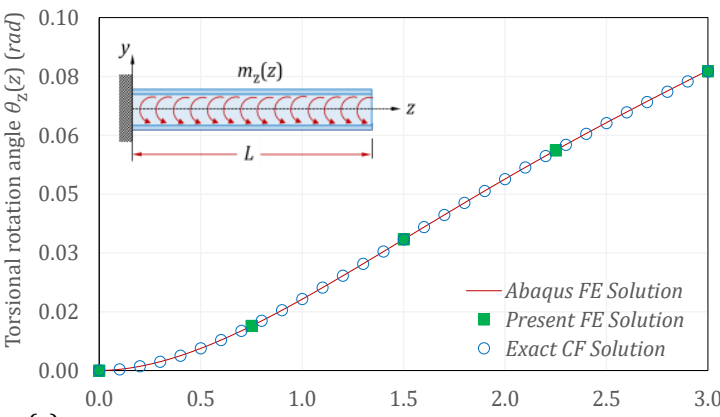

(a)

Beam coordinate $z(m)$

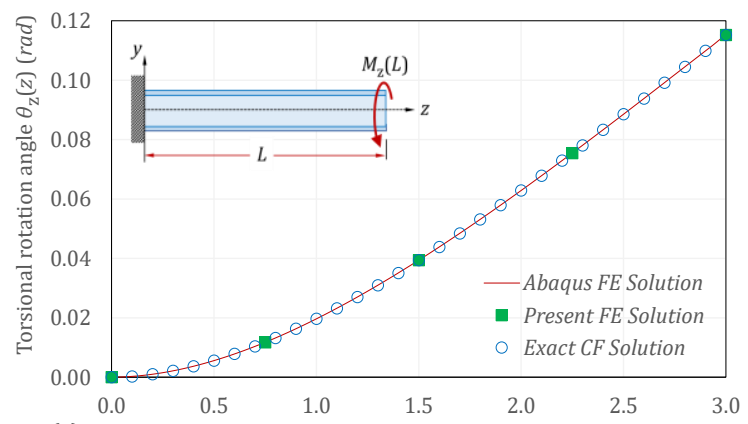

(c)

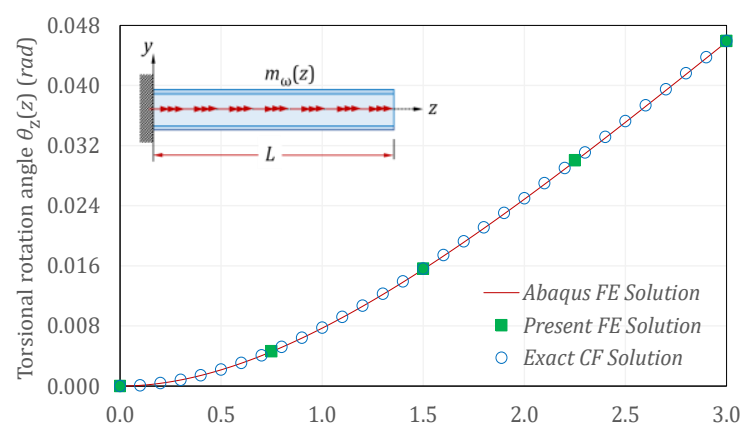

(e)

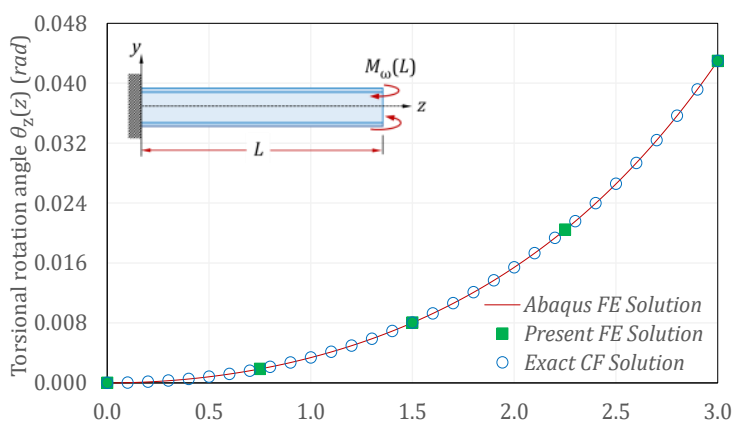

(g)

Beam coordinate $z(m)$

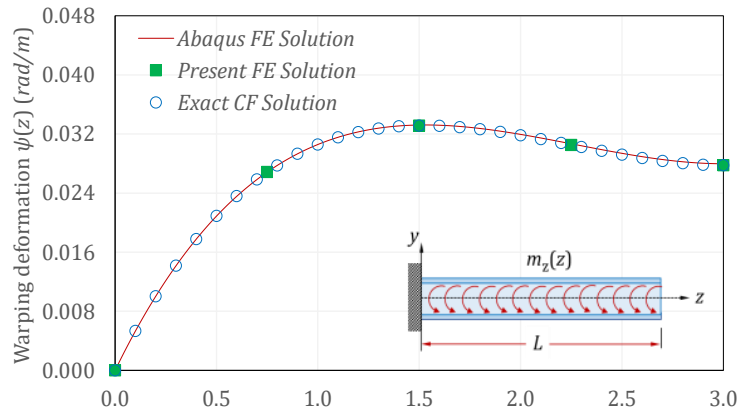

(b)

Beam coordinate $z(m)$

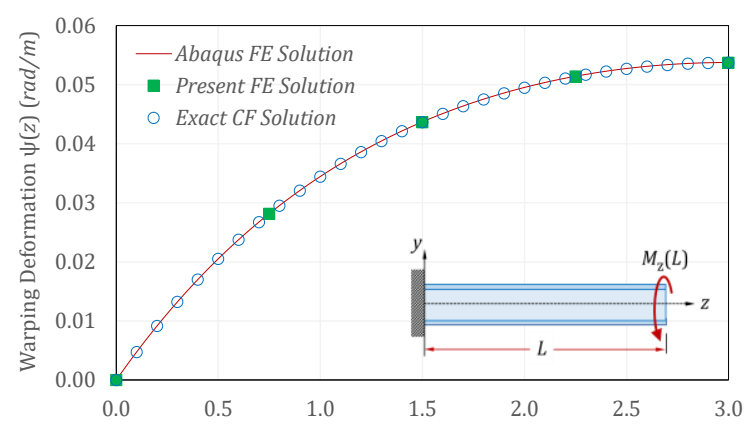

(d)

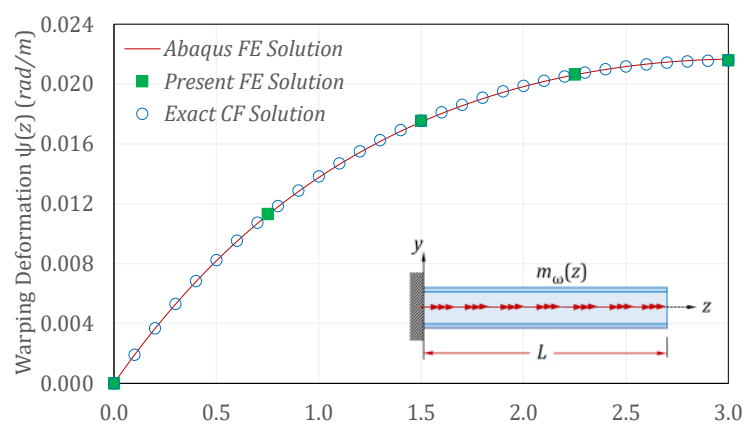

(f)

Beam coordinate $z(m)$

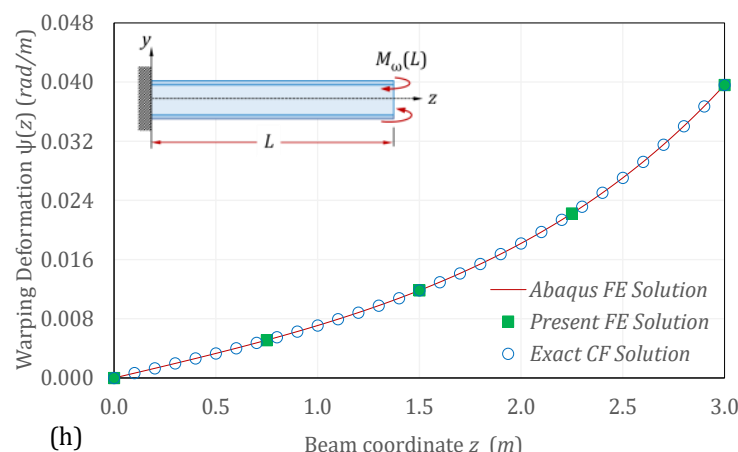

(h)

Fig. 4. Static torsional-warping coupling analysis of cantilever thin-walled I-beam under various twisting and warping moments 

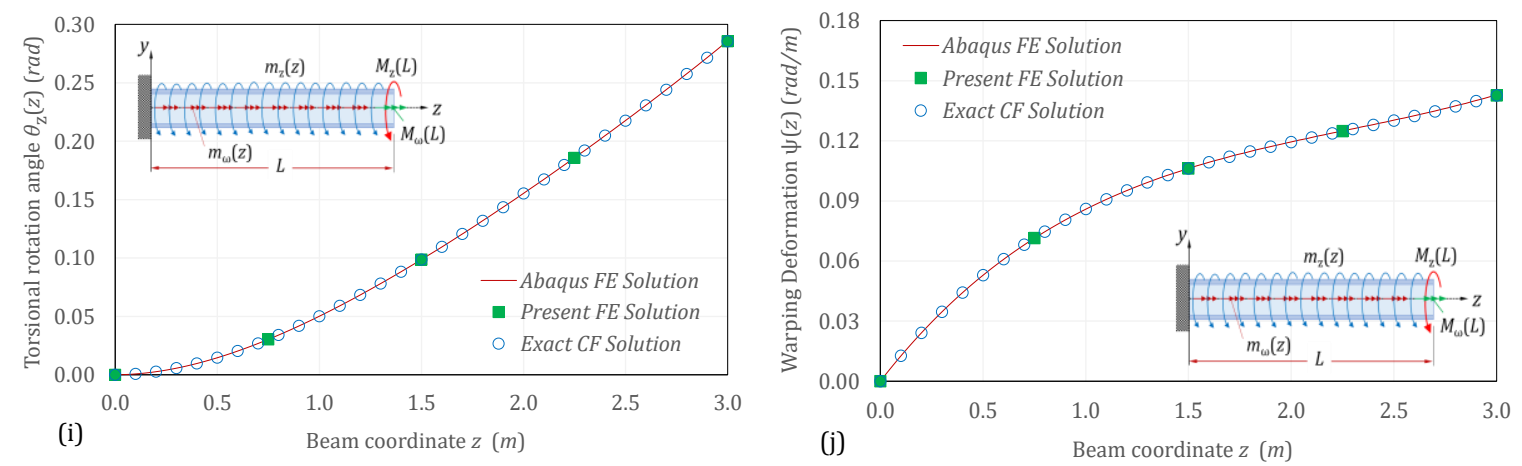

Fig. 4. Continued

\subsection{Example 2 - Fork-supported beam under torsional moments}

A $6 \mathrm{~m}$ thin-walled simply supported I-beam with fork-type end supports subjected to uniformly distributed torsional loading $m_{z}(z)=1.2 \mathrm{kNm} / \mathrm{m}$ and distributed warping moment $m_{\omega}=1.4 \mathrm{kNm}^{2} / \mathrm{m}$ is considered as shown in Fig. 5. The beam is unrestrained along its length except at each beam end where the fork support prevents the crosssection from torsional rotation and moving laterally but allows for the warping. This example is provided to compute the static response for coupled torsional-warping behavior of the given simply supported beam. The material of thin-walled beam is steel with $E=210 \mathrm{GPa}$, and $G=80 \mathrm{GPa}$, while the geometrical properties of the cross-section are illustrated in Table 3. For the sake of validation, the numerical results calculated from the finite element solution (FES) developed in the present study are compared with the ABAQUS finite beam B31OS element model and exact solution in [24].

The static analysis for coupled torsionalwarping response of fork-supported I-beam subjected to uniform distributed twisting moment $m_{z}(z)=1.2 \mathrm{kNm} / \mathrm{m}$ and warping moment $m_{\omega}=1.4$ $\mathrm{kNm}^{2} / \mathrm{m}$ is computed using three different solutions: (a) the exact closed-form solution presented in [24], (b) the finite element solution using four beam elements (10 DOFs), and (c)
ABAQUS finite element model using 100 beam B31OS elements (707 DOFs). Even though, the present finite element formulation based on a single beam element (4 DOFs) provided excellent results but for the sake of comparison four beam elements with 10 DOFs were used.

The static results for nodal torsional rotation $\theta_{z}(z)$ and warping deformation function $\psi(z)$, as illustrated in Fig.6, based on present finite element solution, ABAQUS beam B31OS model, and exact solution developed in [24] are overlaid on the same diagrams for comparison. As a general remark, Fig. 6 shows excellent agreement between all three solutions. Furthermore, the developed finite element results based on four beam elements shows excellent agreement with those based on the ABAQUS finite model solution using 100 beam B31OS elements (707 DOFs).

Table 3. Geometric and properties of doubly symmetric thin-walled I-section beam

\begin{tabular}{ll}
\hline$A$ & $6500 \mathrm{~mm}^{2}$ \\
$\mathrm{I}_{\mathrm{xx}}$ & $45.25 \times 10^{6} \mathrm{~mm}^{4}$ \\
$\mathrm{I}_{\mathrm{yy}}$ & $10.25 \times 10^{6} \mathrm{~mm}^{4}$ \\
$\mathrm{~J}$ & $421.7 \times 10^{3} \mathrm{~mm}^{4}$ \\
$\mathrm{I}_{\omega}$ & $87.62 \times 10^{9} \mathrm{~mm}^{6}$ \\
$\mathrm{D}_{\omega \omega}$ & $41.07 \times 10^{6} \mathrm{~mm}^{4}$ \\
\hline
\end{tabular}



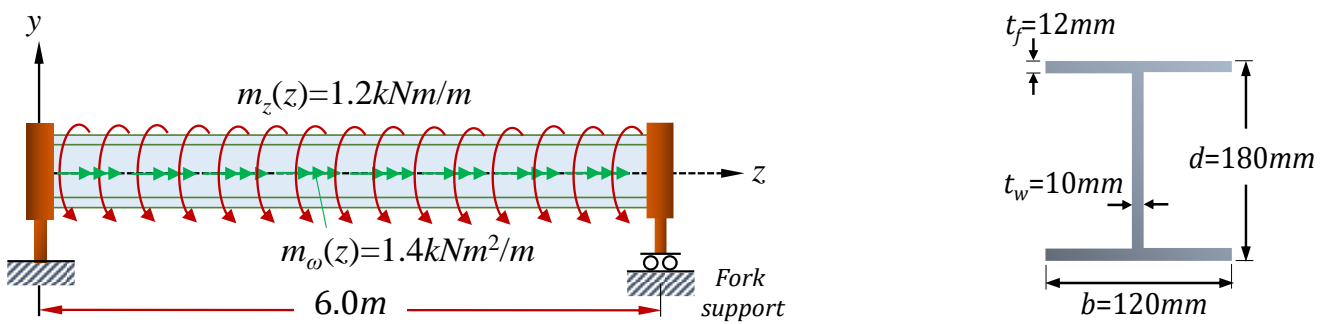

Fig. 5. A Fork-supported I-beam under distributed twisting moment and axial force
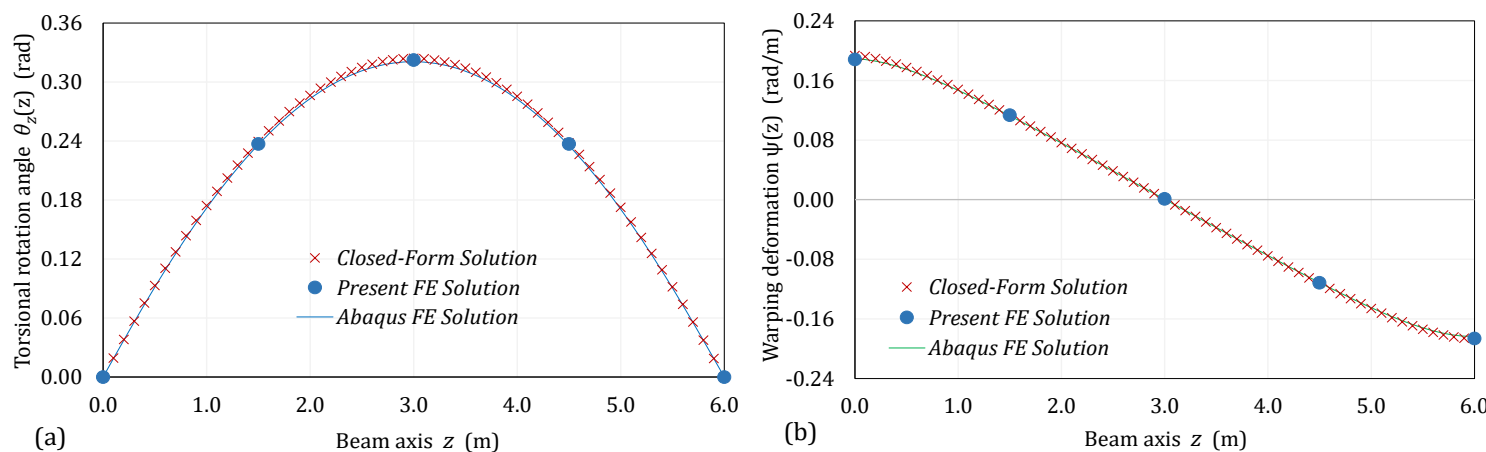

Fig. 6. Static analysis for torsional-warping coupled response of fork-supported thin-walled I-beam under distributed twisting moment

\subsection{Example 4-Validation of finite element formulation}

A fixed-fork thin-walled doubly symmetric Isection of $5 \mathrm{~m}$ length with the same cross-section as given in Example 2 is subjected to various torsional and warping moments; distributed twisting moment $m_{z}(z)=0.5 \mathrm{kNm} / \mathrm{m}$ and distributed warping moment $m_{\omega}=0.8 \mathrm{kNm} 2 / \mathrm{m}$ along beam span, concentrated twisting moments $M_{z 1}(z=1.25 \mathrm{~m})=$ $1.0 \mathrm{kNm}$ and $M_{z 2}(z=3.75 \mathrm{~m})=2.5 \mathrm{kNm}$ applied as shown in Fig. 7. The geometric properties of the beam section are provided in Table 3 . It is required to assess the accuracy and efficiency of the present finite element formulation solution in determining the static response of torsional warping coupled behavior of the beam.

Two solutions are provided for the given beam. The first solution is based on ABAQUS finite element model of 200 beam B31OS elements in which a total of 1,407 degrees of freedom were needed to eliminate the mesh discretization errors and achieve the required accuracy. The second solution is based on the present finite element formulation, in which the beam is subdivided into only four beam elements along the beam span, i.e., the model has only 10 DOFs.

The nodal torsional rotation angle $\theta_{z i}$ and warping deformation function $\psi_{i}$ (for $i=1,2,3, \ldots ., 10)$ are provided in Figs. $8 \mathrm{a}$ and $8 \mathrm{~b}$, respectively, for the static torsional-warping coupled response of the given beam. It is observed form the figures that, the nodal torsional rotation and warping deformation functions predicted by the present finite element model using four beam elements provide an excellent agreement with those based on ABAQUS finite beam solution using 200 beam B31OS elements at a fraction of the computational and modelling cost. Again, this is a natural outcome of the fact that the present finite element formulation is based on the exact shape functions which in turn eliminates discretization errors encountered in finite element formulations. 


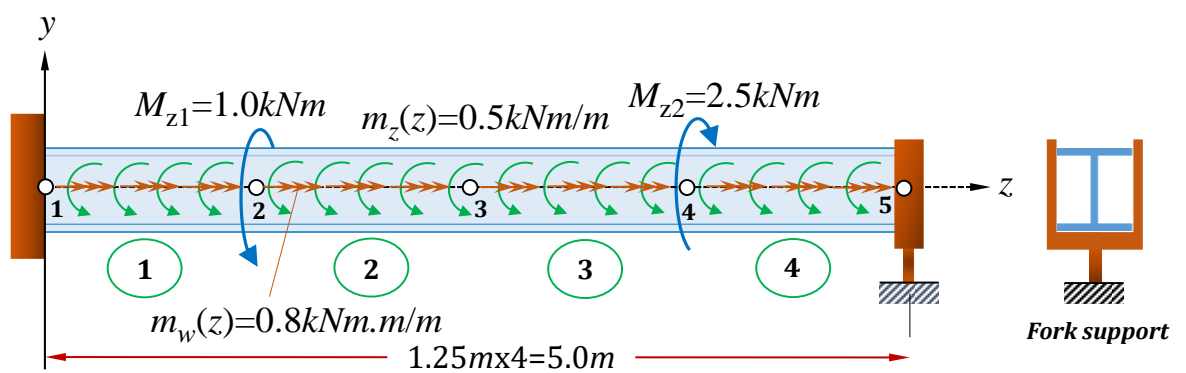

Fig. 7. A fixed-fork thin-walled I-beam under various twisting and warping moments
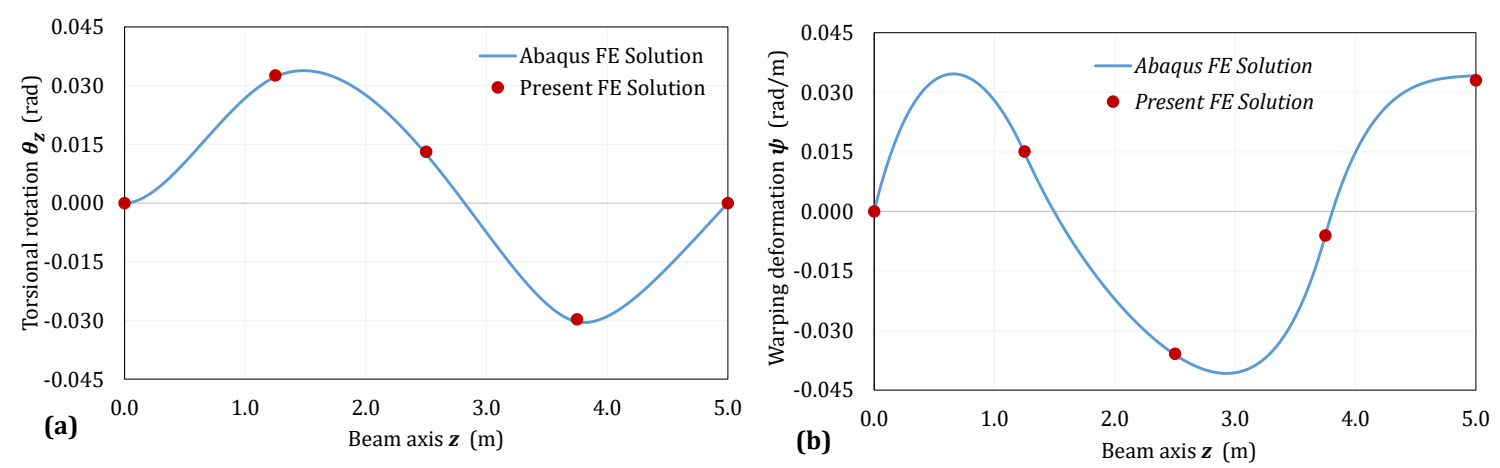

Fig. 8. Static torsional-warping coupled analysis of fixed-fork I-beam under various twisting and warping moments

\section{Conclusions}

1. A super-convergent finite element formulation was developed for open thin-walled beams with doubly symmetric sections. The two-noded beam element is based on shape functions which exactly satisfy the homogeneous form of the static equilibrium torsional-warping coupled equations.

2. The new beam element involves no discretization errors encountered under other interpolation schemes and generally exhibits excellent results while keeping the number of degrees of freedom a minimum.

3. The present finite element solution provides excellent agreement with ABAQUS finite B31OS beam elements at a fraction of the computational and modeling effort.

4. The finite element formulation successfully captures the coupled torsional-warping response of open thin-walled beam with doubly symmetric cross-sections under various twisting and warping moments.

\section{Declaration of conflicting interests}

The author(s) declared no potential conflicts of interest with respect to the research, authorship, and/or publication of this article.

\section{References}

[1] Vlasov VZ (1961) Thin-Walled Elastic Beams. $2^{\text {nd }}$ edition, Jerusalem-Israel Program for Scientific Transactions.

[2] Timoshenko SP, Gere JM (1961) Theory of Elastic Stability. $2^{\text {nd }}$ edition. McGraw-Hill, New York.

[3] Gjelsvik A (1981) The Theory of Thin-Walled Bars. Wiley, New York.

[4] Seaburg PA, Carter CJ (1997) Torsional analysis of structural steel members. American Institute of Steel Construction (AISC), Chicago.

[5] Back SY, Will KM (1998) A shear-flexible element with warping for thin-walled open beams. International Journal for Numerical Methods in Engineering 43(7):1173-1191.

[6] Mohareb M, Nowzartash F (2002) Exact finite element for non-uniform torsion of open sections. Journal of Structural Engineering 129(2):215-223. 
[7] Sapountzakis EJ, VG Mokos VG (2003) Warping shear stresses in non-uniform torsion by BEM. Computational Mechanics 30(2):131-142.

[8] Kim NI, Kim MY (2005) Exact dynamic/static stiffness matrices of non-symmetric thin-walled beams considering coupled shear deformation effects. Thin-Walled Structures 43(5):701-743.

[9] Pavazza R (2005) Torsion of thin-walled beams of open cross-section with influence of shear. International Journal of Mechanical Sciences 47(7):1099-1122.

[10] Erkmen ER, Mohareb M (2006) Torsion analysis of thin-walled beams including shear deformation effects. Thin-Walled Structures 44(10):1096-1108.

[11] El Fatmi RE (2007) Non-uniform warping including the effects of torsion and shear forces. Part I-A general beam theory. International Journal of Solids and Structures 44(18-19):5912-5929.

[12] El Fatmi RE (2007) Non-uniform warping including the effects of torsion and shear forces. Part II-Analytical and numerical applications. International Journal of Solids and Structures 44(18-19):5930-5952.

[13] Campanile A, Mandarino M, Piscopo V (2009) On the exact solution of non-uniform torsion for beams with asymmetric cross-section. World Academy of Science, Engineering and Technology 3(7):46-53.

[14] Mokos VG, Sapountzakis EJ (2011) Secondary torsional moment deformation effect by BEM. International Journal of Mechanical Sciences 53(10):897-909.

[15] Wang ZQ, Zhao JC, Zhang DX, Gong JH (2012) Restrained torsion of open thin-walled beams including shear deformation effects. Journal of Zhejiang University, Science A (Applied Physics and Engineering) 13(4):260-273.

[16] Sapountzakis EJ (2013) Bars under torsional loading- a generalized beam theory approach. Civil Engineering, Hindawi Publishing Corporation Article ID 916581.
[17] Pavazza R, Matokovic A, Plazibat B (2013) Torsion of thin-walled beams of symmetrical open cross-sections with influence of shear. Transactions of Famena XXXVII-2.

[18] Aminbaghai M, Murin J, Balduzzi G, Hrabovsky J, Hochreiner G, Mang H (2017) Second-order torsional warping theory considering the secondary torsion-moment deformation-effect. Engineering Structures 147:724-739.

[19] Mechab I, El-Meiche N, Bernard F (2017) Analytical study for the development of a new warping function for high order beam theory. Composites Part B: Engineering 119:18-31.

[20] Nguyen TC, Tang VL, Nguyen TS, Nguyen QT, Huynh TP (2019), Analysis of thin-walled bars stress state with an open section, IOP Conference, Series-Materials Science and Engineering, 661:012011.

[21] Murin J, Aminbaghai M, Hrabovsky J, Balduzzi G, Dorn M, Mang H (2018) Torsional warping eigenmodes of FGM beams with longitudinally varying material properties. Engineering Structures 175:912-925.

[22] Aribas U, Ermis M, Eratli N, Omurtag M (2019) The static and dynamic analyses of warping included composite exact conical helix by mixed FEM. Composite Part B: Engineering 160:285297.

[23] Aribas U, Ermis M, Kutlu A, Eratli N, Omurtag M (2020) Forced vibration analysis of composite geometrically exact elliptical cone helices via mixed FEM. Mechanics of Advanced Materials and Structures (in press).

[24] Hjaji MA, Nagiar HM, Allaboudi EG, Krer MM (2021) Torsional analysis of open thin-walled doubly symmetric beams under torsion and bimoment. Journal of Mechanical Engineering (accepted). 